\title{
Discrete-Time System Conditional Optimization in the Parameter Space with Nonzero Initial Conditions
}

\author{
Vladimir ZARIĆ*, Zoran BUČEVAC, Radiša JOVANOVIĆ
}

\begin{abstract}
The paper presents a new approach to the design of the classical PD controller for the plant in the closed loop control system. Proposed controller has two unknown adjustable parameters which are designed by the algebraic method in the two dimensional parameter space, by using the newly discovered characteristic polynomial of the row nondegenerate full transfer function matrix. The system relative stability, in regard to the chosen damping coefficient is achieved. The optimality criterion is the minimal value of the performance index and sum of squared error is taken as an objective function. The output error used in the performance index is influenced by all actions on the system at the same time: by nonzero initial conditions and by nonzero inputs. In the classical approach the output error is influenced only by nonzero inputs. Experimental results confirm that by taking into account nonzero initial conditions, an optimal solution is obtained, which is not the case with the classical method where optimization is performed at zero initial conditions.
\end{abstract}

Keywords: conditional optimization; discrete-time control systems; full transfer function matrix; nonzero initial conditions; relative stability; two parameters synthesis

\section{INTRODUCTION}

The system full transfer function matrix enables to treat the simultaneous actions of initial conditions and of the input vector. Usually, the region of poles location was determined by demanded damping coefficient or/and settling time.

Pioneering work on the problem in the parameter plane was conducted by Vishnegradski [2] and Neymark [3]. Dušan Mitrović [4-6] developed grapho-analytical method for analysis and synthesis of linear control systems where only the last two coefficients of characteristic polynomial are considered as varying. Mitrović's method was generalized firstly by Siljak who permitted any two characteristic polynomial coefficients to be adjustable parameters [7], and just afterwards Šiljak [8] and Grujić [9] considered some characteristic polynomial coefficients to be linearly dependent on two adjustable parameters. Šiljak presented a method that enables the designer to obtain information about the effects on the absolute and relative stability of two adjusting system parameters where all characteristic polynomial coefficients are linearly dependent on those two adjusting parameters, $[10,11]$. He established the concept of conditional optimization in the parameter plane by introducing one extra criterion for the system, in addition to its relative stability, which is the minimal value of the performance index in the form of the integral of squared errors, [8]. This was just designated as the conditional parameter optimization. In that way, the system was relatively stable with the desired damping coefficient or/and settling time, and had the finer transient state behavior regarding, e.g., overshoot. Grujić went further in $[12,13]$ to permit denominator and numerator polynomial coefficients of a system transfer function to be dependent linearly on two unknown adjustable parameters. He gathered together conditional optimization and another aspect proposed by Rakic [14], the system impulse response through the numerical values calculation of its transfer function residues.

Quite in a different way, approximately in the same period of time, Kalman made a great progress in the discrete-time linear systems optimization $[15,16]$. He developed a matrix synthesis method of the controller algorithm:
$\boldsymbol{u}(k)=-\boldsymbol{K} \boldsymbol{x}(k), \boldsymbol{K} \in \mathbb{R}^{m \times n}$

By means of Pontryagin's maximum principle and Bellman's continuous dynamic programming. His method does not have constraints to the number of the matrix gain $\boldsymbol{K}$ coefficients, that is, the number of controls and state variables. Kalman's great contribution is that his method is matrix synthesis of all controller parameters at the same time.

To the present day plant control by the classical control algorithm has remained very actual and researchers are dealing with it all the time. Xu et al. [17] determined stabilizing PID gains region in the parameter space for a digital system on the basis of pre-developed results for continuous time systems. Keel et al. [18] have given a complete solution in the sense that a constructive yes or no answer to whether stabilization is possible, is given and, in case it is possible, the entire set is determined by solving sets of linear inequalities in two variables obtained by gridding over the third variable. An effective computational procedure to determine all first order controllers that stabilize a given discrete-time LTI system is described in Tantaris et al. [19]. Li et al. [20] have provided a graphical method to determine the complete set of all stabilizing PID-type controllers in the parameter space by analytically determining stabilizing boundaries using the D-decomposition technique. Gryazinaand Polyak [21] extend D-decomposition for the matrix case, i.e. for MIMO systems. That technique can be helpful for design of low-order controllers and for detailed robustness analysis. Gryazina and Polyak in [22] provided an extension of D-decomposition method for polynomials. Other authors considered optimal PID controller tuning. Padula and Visioli [23] used standard and fractional-order PID controllers. They showed that the use of fractionalorder integral action is not advantageous, while the use of a fractional-order derivative action provides a performance improvement. Barbosa and Jesus [24] considered fractional order PID controller and metaheuristic tuning algorithm Cuckoo Search, inspired by living beings behavior in nature. Their results revealed that the Cuckoo Search can outperform some state of the art stochastic algorithms, such as the widely used genetic algorithms. 
The results based on all mentioned approaches, except Kalman's, were developed under decades-long controversy between the stability concept and the classical system transfer function. The system stability is by definition the dynamical property of the system in the free working mode under all zero inputs and arbitrary initial conditions. On the other side the transfer function is defined for the system in the forced working regime under nonzero input and all zero initial conditions. This controversy has been recently solved by introducing and developing the full transfer function matrix $[1,25,26]$. In this paper the conditional optimization synthesis procedure is carried out by the characteristic polynomial of the full transfer function matrix. That characteristic polynomial in the general case does not have to be the same as the characteristic polynomial of the classical transfer function as is showed in [1]. The characteristic polynomial of the row non degenerate full transfer function matrix $[1,25,26]$ is the only adequate and appropriate to be used for the objective test of the system stability and optimization.

We use a new form of the performance index in order to design controller to cope with the real working conditions. It is the sum of the squared errors occurring in the most general and realistic circumstances, under simultaneous influence of the external nonzero inputs and the nonzero initial conditions.

Both Kalman's regulator and conditional optimization have their advantages and disadvantages. A significant progress in the proposed new conditional optimization is that the controller is dynamic vs static Kalman's regulator. To get the matrix gain $\boldsymbol{K}$ for Kalman's regulator, it is necessary to solve the matrix nonlinear Riccati equation, which is an aggravating circumstance, despite the present age of digital computers. Furthermore, related to Kalman's regulator, the right choice of matrices $Q$ and $R$ is not easy. Estimate procedures are to be applied when the state variables are not measurable. Conditional optimization provides relative stability whereas Kalman's method does not. One part of Kalman's optimality criterion concerns process quality and another one the energy consumption. In this way the result is a compromise between the two requirements. Our dynamic controller optimality criterion vs Kalman's one, fully concerns process quality, which leads to a higher degree of process quality than in Kalman's case.

Relative stability, with respect to the desired damping coefficient is carried out by using the characteristic polynomial of the full transfer function matrix which in the general case does not have to match the classical one, [1]. The choice of optimal parameters in the parameter plane yields the fine transient state. It is carried out by means of the new performance index characteristic. The calculated performance index values are related to the constant desired damping coefficient.

Illustrative example is given to show the difference in the system behavior, when it is designed in the classical and proposed novel way. The differences are detected by a practical experiment.

\section{PROBLEM STATEMENT}

\subsection{Plant}

Design procedure has been applied and tested experimentally on a DC servomotor with gear and load. The experiments were performed using a Quanser rotary servomotor, SRV02. This model is equipped with the optical encoder and tachometer for measuring motor position and speed, respectively. The Q8-USB data acquisition board was used for real-time data acquisition and control, in the Windows environment, with Matlab/Simulink and QUARC@, real-time control software.

At first, a $1,3 \mathrm{~s}$ long or $650 \mathrm{k}$ servo motor works in open loop to get the initial conditions and afterwards to be switched to the closed loop operation mode. During that first 1,3 s nominal input voltage is $1,5 \mathrm{~V}$. At the exact moment of $1,3 \mathrm{~s}$, a unit step voltage was brought to the plant to identify its mathematical model by velocity, since that is a first order plant. The mathematical model of the plant by position is simply obtained by integrating the mathematical model by velocity. Model verification of plant's discrete model is shown in Fig. 1.

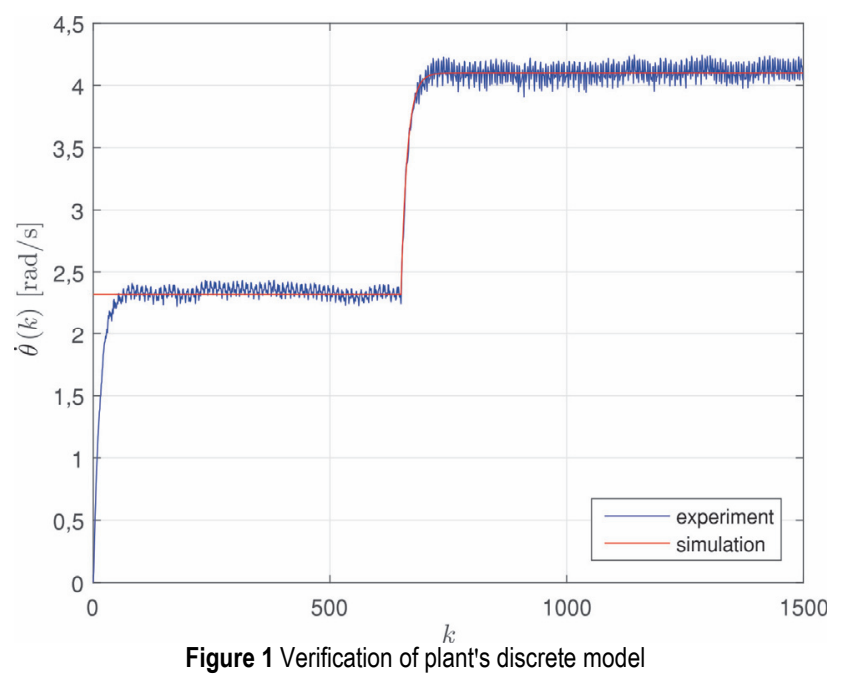

Choosing $y=\theta$ and $u_{\mathrm{p}}=U$, an identified discretetime input-output (IO) model of the plant for the sampling $\operatorname{period} T=0,002 \mathrm{~s}$ is:

$$
\begin{aligned}
& y(k+2)-1,9311 y(k+1)+0,9311 y(k)= \\
& =0,0001242 u_{\mathrm{p}}(k+1)+0,0001212 u_{\mathrm{p}}(k)
\end{aligned}
$$

The linear time-invariant discrete-time of the most general SISO plant is considered and it is described by its input-output equation,

$$
\begin{aligned}
& a_{v \mathrm{P}} y(k+v)+a_{(v-1) \mathrm{P}} y(k+v-1)+\cdots+a_{1 \mathrm{P}} y(k+1)+ \\
& +a_{0 \mathrm{P}} y(k)=b_{0 \mathrm{P}} u_{\mathrm{P}}(k)+\cdots+b_{(\mu-1) \mathrm{P}}(k+\mu-1)+ \\
& +b_{\mu}(k+\mu), a_{v \mathrm{P}}=1, \eta \in \mathbb{N}, \mu \in \mathbb{N}_{0}, \mu \leq v,
\end{aligned}
$$

where $a_{j \mathrm{P}} \in \mathbb{R}, \forall j=0,1,2, \cdots, v$ and $b_{j \mathrm{P}} \in \mathbb{R}$,

$\forall j=0,1,2, \cdots, \mu$ are real numbers and $k \in \mathbb{N}_{0}$, $y(k+j) \in \mathbb{R}$ is the plant output at time $k+j$, $\forall j=0,1,2, \cdots, v, u_{\mathrm{P}}(k+j) \in \mathbb{R}$ is the plant input at time $k+j, \forall j=0,1,2, \cdots, \mu, \mu \leq \eta$.

The compact form of Eq. (2) is as follows $[1,26]$ 
$\boldsymbol{A}_{\mathrm{P}}^{(2)} \boldsymbol{y}^{2}=\boldsymbol{B}_{\mathrm{P}}^{(1)} \boldsymbol{u}_{\mathrm{P}}^{1}$

$\boldsymbol{A}_{\mathrm{P}}^{(2)}=\left[\begin{array}{lll}0,9311 & -1,9311 & 1\end{array}\right]$,

$\boldsymbol{B}_{\mathrm{P}}^{(1)}=\left[\begin{array}{ll}0,0001212 & 0,0001242\end{array}\right]$,

$\boldsymbol{y}^{2}=\left[\begin{array}{lll}y(k) & y(k+1) & y(k+2)\end{array}\right]^{\mathrm{T}}$,

$\boldsymbol{u}_{\mathrm{P}}^{1}=\left[\begin{array}{ll}u_{\mathrm{P}}(k) & u_{\mathrm{P}}(k+1)\end{array}\right]^{\mathrm{T}}$.

\subsection{Controller}

The general form of the first order PD controller is,

$T_{1} \frac{\Delta u_{\mathrm{p}}(k)}{T}+u_{\mathrm{p}}(k)=K \varepsilon(k)+K_{\mathrm{d}} \frac{\Delta \varepsilon(k)}{T}$.

By selecting the sample period $T=0,002$ and $T_{1}=1$ we obtain,

$u_{\mathrm{p}}(k+1)-0,998 u_{\mathrm{p}}(k)=$

$=\left(0,002 K-K_{\mathrm{d}}\right) \varepsilon(k)+K_{\mathrm{d}} \varepsilon(k+1)$.

The compact form of Eq. (6) is as follows $[1,26]$,

$\boldsymbol{A}_{\mathrm{C}}^{(1)} \boldsymbol{u}_{\mathrm{P}}^{1}=\boldsymbol{B}_{\mathrm{C}}^{(1)} \boldsymbol{\varepsilon}^{1}$,

$\boldsymbol{A}_{\mathrm{C}}^{(1)}=\left[\begin{array}{ll}-0,998 & 1\end{array}\right]$,

$\boldsymbol{B}_{\mathrm{C}}^{(1)}=\left[0,002 K-K_{\mathrm{d}} \quad K_{\mathrm{d}}\right]$,

$\boldsymbol{u}_{\mathrm{P}}^{1}=\left[\begin{array}{ll}u_{\mathrm{P}}(k) & u_{\mathrm{P}}(k+1)\end{array}\right]^{\mathrm{T}}$,

$\varepsilon^{1}=\left[\begin{array}{ll}\varepsilon(k) & \varepsilon(k+1)\end{array}\right]^{\mathrm{T}}$.

\subsection{The Overall Closed Loop System}

The overall closed loop system is described in the compact form by:

$\boldsymbol{A}^{(3)}(\alpha, \beta) \boldsymbol{y}^{3}(k)=$

$=\boldsymbol{B}^{(2)}(\alpha, \beta) \boldsymbol{i}^{2}(k), \forall k \in N_{0}, \alpha=K, \beta=K_{\mathrm{d}}$,

$\boldsymbol{y}^{3}(k)=\left[\begin{array}{llll}y(k) & y(k+1) & y(k+2) & y(k+3)\end{array}\right]^{\mathrm{T}}$,

$\boldsymbol{i}^{2}(k)=\left[\begin{array}{lll}\boldsymbol{i}^{\mathrm{T}}(k) & \boldsymbol{i}^{\mathrm{T}}(k+1) & \boldsymbol{i}^{\mathrm{T}}(k+2)\end{array}\right]^{\mathrm{T}}$,

$\boldsymbol{i}=\left[\begin{array}{ll}r & d\end{array}\right]^{\mathrm{T}}$,

$\boldsymbol{A}^{(3)}(\alpha, \beta)=$

$=\left[\begin{array}{llll}a_{0}(\alpha, \beta) & a_{1}(\alpha, \beta) & a_{2}(\alpha, \beta) & a_{3}(\alpha, \beta)\end{array}\right]=$

$=\left[\begin{array}{c}2,4250 \cdot 10^{-7} \alpha-1,2125 \cdot 10^{-4} \beta-0,9292 \\ 2,4834 \cdot 10^{-7} \alpha-2,9214 \cdot 10^{-6} \beta+2,8583 \\ 1,2417 \cdot 10^{-4} \beta-2,9291 \\ 1\end{array}\right]^{\mathrm{T}}$,

$$
\begin{aligned}
& \boldsymbol{B}^{(2)}(\alpha, \beta)=\left[\begin{array}{lll}
\boldsymbol{B}_{0}(\alpha, \beta) & \boldsymbol{B}_{1}(\alpha, \beta) \quad \boldsymbol{B}_{2}(\alpha, \beta)
\end{array}\right]= \\
& =\left[\begin{array}{c}
{\left[\begin{array}{cc}
2,4250 \cdot 10^{-7} \alpha-1,2125 \cdot 10^{-4} \beta & -1,2101 \cdot 10^{-4}
\end{array}\right]^{\mathrm{T}}} \\
{\left[\begin{array}{cc}
2,4834 \cdot 10^{-7} \alpha-2,9214 \cdot 10^{-6} \beta & -2,6731 \cdot 10^{-6}
\end{array}\right]^{\mathrm{T}}} \\
{\left[\begin{array}{cc}
1,2417 \cdot 10^{-4} \beta & 1,2417 \cdot 10^{-4}
\end{array}\right]^{\mathrm{T}}}
\end{array}\right]^{\mathrm{T}}
\end{aligned}
$$

The input-output (IO) system's, given by Eq. (8), full transfer function matrix $\boldsymbol{F}(z)$ reads [1],

$$
\begin{aligned}
& \boldsymbol{F}(z)=\boldsymbol{F}_{\mathrm{D}}^{-1}(z) \boldsymbol{F}_{\mathrm{N}}(z)=\left[\boldsymbol{A}^{(3)}(\cdot) \boldsymbol{S}_{1}^{(3)}(z)\right]^{-1} . \\
& \cdot\left[\begin{array}{lll}
\boldsymbol{B}^{(2)}(\cdot) \boldsymbol{S}_{2}^{(2)}(z) & -\boldsymbol{B}^{(2)}(\cdot) \boldsymbol{Z}_{2}^{(2)}(z) & \boldsymbol{A}^{(3)}(\cdot) \boldsymbol{Z}_{1}^{(3)}(z)
\end{array}\right] \text {, } \\
& \boldsymbol{S}_{1}^{(3)}(z)=\left[\begin{array}{llll}
1 & z & z^{2} & z^{3}
\end{array}\right]^{\mathrm{T}} \text {, } \\
& \boldsymbol{S}_{2}^{(2)}(z)=\left[\begin{array}{cccccc}
1 & 0 & z & 0 & z^{2} & 0 \\
0 & 1 & 0 & z & 0 & z^{2}
\end{array}\right]^{\mathrm{T}}, \\
& \boldsymbol{Z}_{2}^{(2)}(z)=\left[\begin{array}{cccc}
0 & 0 & 0 & 0 \\
0 & 0 & 0 & 0 \\
z & 0 & 0 & 0 \\
0 & z & 0 & 0 \\
z^{2} & 0 & z & 0 \\
0 & z^{2} & 0 & z
\end{array}\right], \boldsymbol{Z}_{1}^{(3)}(z)=\left[\begin{array}{ccc}
0 & 0 & 0 \\
z & 0 & 0 \\
z^{2} & z & 0 \\
z^{3} & z^{2} & z
\end{array}\right] .
\end{aligned}
$$

$$
\begin{aligned}
\boldsymbol{F}(z)= & \frac{1}{z^{3}+\left(\begin{array}{l}
1,2417 \cdot 10^{-4} \beta+ \\
-2,9291
\end{array}\right) z^{2}+\left(\begin{array}{l}
2,4834 \cdot 10^{-7} \alpha+ \\
-2,9214 \cdot 10^{-6} \beta+ \\
+2,8583
\end{array}\right) z+} . \\
& +\left(2,4250 \cdot 10^{-7} \alpha-1,2125 \cdot 10^{-4} \beta-0,9292\right)
\end{aligned} .
$$

$$
\left[\begin{array}{c}
1,2417 \cdot 10^{-4} \beta z^{2}+\left(2,4834 \cdot 10^{-7} \alpha-2,9214 \cdot 10^{-6} \beta\right) z+ \\
+2,4250 \cdot 10^{-7} \alpha-1,2125 \cdot 10^{-4} \beta \\
1,2417 \cdot 10^{-4} z^{2}-2,6730 \cdot 10^{-6} z-1,2101 \cdot 10^{-4} \\
-1,2417 \cdot 10^{-4} \beta z^{2}-\left(2,4834 \cdot 10^{-7} \alpha-2,9214 \cdot 10^{-6} \beta\right) z \\
-1,2417 \cdot 10^{-4} z^{2}+2,6730 \cdot 10^{-6} z \\
-1,2417 \cdot 10^{-4} \beta z \\
-1,2417 \cdot 10^{-4} z \\
z^{3}+\left(1,2417 \cdot 10^{-4} \beta-2,9291\right) z^{2}+ \\
+\left(2,4834 \cdot 10^{-7} \alpha-2,9214 \cdot 10^{-6} \beta+2,8583\right) z \\
z^{2}+\left(1,2417 \cdot 10^{-4} \beta-2,9291\right) z \\
z
\end{array}\right]
$$

\subsection{Relative Stability}

The full transfer function matrix is row nondegenerate, which implies that the system characteristic polynomial is: 


$$
\begin{aligned}
& z^{3}+\left(\begin{array}{l}
1,2417 \cdot 10^{-4} \beta+ \\
-2,9291
\end{array}\right) z^{2}+\left(\begin{array}{l}
2,4834 \cdot 10^{-7} \alpha+ \\
-2,9214 \cdot 10^{-6} \beta+ \\
+2,8583
\end{array}\right) z+ \\
& +\left(2,4250 \cdot 10^{-7} \alpha-1,2125 \cdot 10^{-4} \beta-0,9292\right) \equiv \\
& \equiv a_{3}(\alpha, \beta) z^{3}+a_{2}(\alpha, \beta) z^{2}+a_{1}(\alpha, \beta) z+a_{0}(\alpha, \beta), \\
& a_{j}(\alpha, \beta)=b_{j} \alpha+c_{j} \beta+d_{j}, j=0,1,2,3 .
\end{aligned}
$$

In order to obtain the loci in the $\alpha \beta$ parameter plane of the constant damping coefficient, we choose the constant value of $\varsigma, 0<\varsigma<1$, change the values of the natural frequency $\omega_{n}$ with a numerical step, and use the following expressions $[10,11]$,

$\alpha=\frac{C_{1} D_{2}-C_{2} D_{1}}{B_{1} C_{2}-B_{2} C_{1}}$

$\beta=\frac{B_{2} D_{1}-B_{1} D_{2}}{B_{1} C_{2}-B_{2} C_{1}}$

where,

$$
\begin{aligned}
& B_{1}\left(\omega_{\mathrm{n}}, \varsigma\right)=2,4834 \cdot 10^{-7} \mathrm{e}^{-0,002 \varsigma \omega_{\mathrm{n}}} . \\
& \cdot \cos \left(0,002 \omega_{\mathrm{n}} \sqrt{1-\varsigma^{2}}\right)+2,4250 \cdot 10^{-7}, \\
& B_{2}\left(\omega_{\mathrm{n}}, \varsigma\right)=2,4834 \cdot 10^{-7} \mathrm{e}^{-0,002 \varsigma \omega_{\mathrm{n}}} . \\
& \cdot \sin \left(0,002 \omega_{\mathrm{n}} \sqrt{1-\varsigma^{2}}\right) \text {, } \\
& C_{1}\left(\omega_{\mathrm{n}}, \varsigma\right)=1,2417 \cdot 10^{-4} \mathrm{e}^{-0,004 \varsigma \omega_{\mathrm{n}}} \text {. } \\
& \cdot \cos \left(0,004 \omega_{\mathrm{n}} \sqrt{1-\varsigma^{2}}\right)+ \\
& -2,9214 \cdot 10^{-6} \mathrm{e}^{-0,002 \varsigma \omega_{\mathrm{n}}} \cos \left(0,002 \omega_{\mathrm{n}} \sqrt{1-\varsigma^{2}}\right)- \\
& -1,2125 \cdot 10^{-4} \text {, } \\
& C_{2}\left(\omega_{\mathrm{n}}, \varsigma\right)=1,2417 \cdot 10^{-4} \mathrm{e}^{-0,004 \varsigma \omega_{\mathrm{n}}} \text {. } \\
& \cdot \sin \left(0,004 \omega_{\mathrm{n}} \sqrt{1-\varsigma^{2}}\right)+ \\
& -2,9214 \cdot 10^{-6} \mathrm{e}^{-0,002 \varsigma \omega_{\mathrm{n}}} \sin \left(0,002 \omega_{\mathrm{n}} \sqrt{1-\varsigma^{2}}\right) \text {, } \\
& D_{1}\left(\omega_{\mathrm{n}}, \varsigma\right)=2,8583 \mathrm{e}^{-0,002 \varsigma \omega_{\mathrm{n}}} \cos \left(0,002 \omega_{\mathrm{n}} \sqrt{1-\varsigma^{2}}\right)+ \\
& -2,9291 \mathrm{e}^{-0,004 \varsigma \omega_{\mathrm{n}}} \cos \left(0,004 \omega_{\mathrm{n}} \sqrt{1-\varsigma^{2}}\right)+ \\
& +\mathrm{e}^{-0,006 \varsigma \omega_{\mathrm{n}}} \cdot \cos \left(0,006 \omega_{\mathrm{n}} \sqrt{1-\varsigma^{2}}\right)-0,9292, \\
& D_{2}\left(\omega_{\mathrm{n}}, \varsigma\right)=2,8583 \mathrm{e}^{-0,002 \varsigma \omega_{\mathrm{n}}} \sin \left(0,002 \omega_{\mathrm{n}} \sqrt{1-\varsigma^{2}}\right)+ \\
& -2,9291 \mathrm{e}^{-0,004 \varsigma \omega_{\mathrm{n}}} \sin \left(0,004 \omega_{\mathrm{n}} \sqrt{1-\varsigma^{2}}\right)+ \\
& +\mathrm{e}^{-0,006 \varsigma \omega_{\mathrm{n}}} \cdot \sin \left(0,006 \omega_{\mathrm{n}} \sqrt{1-\varsigma^{2}}\right) .
\end{aligned}
$$

The loci in the $\alpha \beta$ parameter plane of the constant damping coefficient $\varsigma=0,7$ is shown in Fig. 2 .

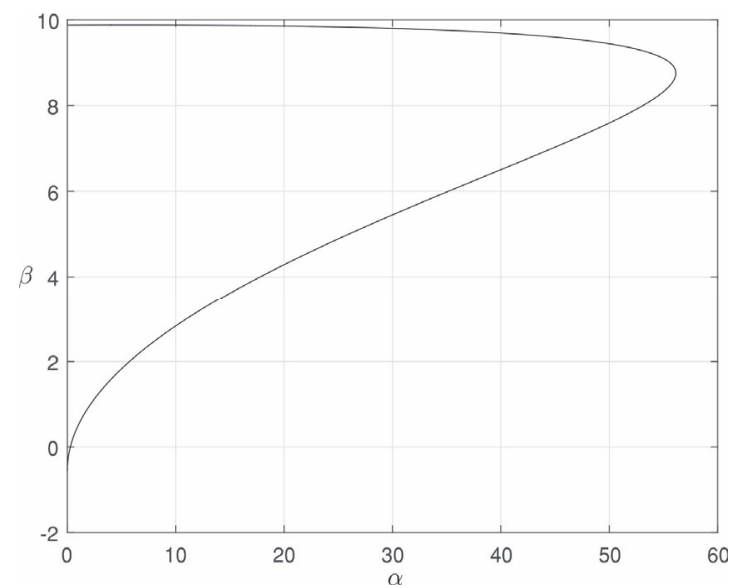

Figure 2 The loci in the $\alpha \beta$ parameter plane of the constant damping coefficient $\zeta=0,7$

\subsection{Performance Index}

The proposed form of performance index $I$ is the sum of the squared output errors:

$$
I=\sum_{k=0}^{\infty} \varepsilon^{2}(k), \varepsilon(k)=r(k)-y(k)
$$

where error $\varepsilon(k)$ occurred under the influence of all actions on the system, external nonzero input and nonzero initial conditions, at the same time. It is more natural and realistic in practice than the error $\varepsilon(k)$ that has been used in literature so far influenced only by nonzero input but for all zero initial conditions.

The full block diagram $[1,26]$ of the system is shown in Fig. 3.

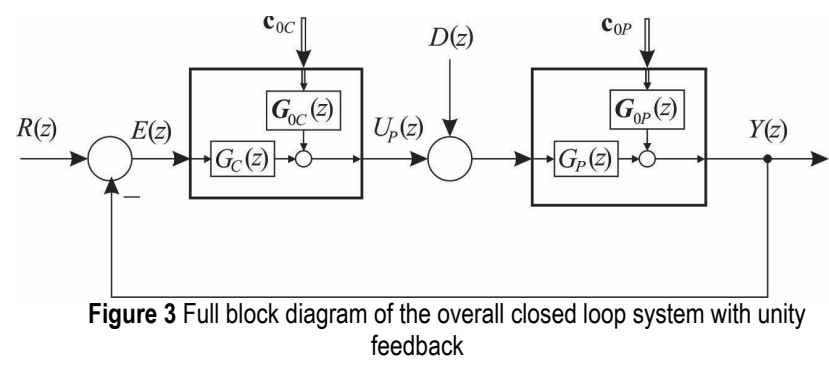

From the block diagram, it is easy to obtain:

$$
\begin{aligned}
& Y(z)=\boldsymbol{F}(z) \boldsymbol{V}(z)=\left[\begin{array}{c}
\frac{G_{\mathrm{P}}(z) G_{\mathrm{C}}(z)}{1+G_{\mathrm{P}}(z) G_{\mathrm{C}}(z)} \\
\frac{G_{\mathrm{P}}(z)}{1+G_{\mathrm{P}}(z) G_{\mathrm{C}}(z)} \\
\frac{G_{\mathrm{P}}(z)}{1+G_{\mathrm{P}}(z) G_{\mathrm{C}}(z)} \boldsymbol{G}_{0 \mathrm{C}}^{\mathrm{T}}(z) \\
\frac{1}{1+G_{P}(z) G_{C}(z)} \boldsymbol{G}_{0 \mathrm{P}}^{\mathrm{T}}(z)
\end{array}\right]^{\mathrm{T}} \\
& \cdot\left[\begin{array}{c}
\boldsymbol{I}(z) \\
\boldsymbol{c}_{0 \mathrm{C}} \\
\boldsymbol{c}_{0 \mathrm{P}}
\end{array}\right]=\left[\begin{array}{ll}
\boldsymbol{G}(z) & \boldsymbol{G}_{0}(z)
\end{array}\right]\left[\begin{array}{c}
\boldsymbol{I}(z) \\
\boldsymbol{c}_{0}
\end{array}\right], \boldsymbol{I}(z)=\left[\begin{array}{c}
R(z) \\
D(z)
\end{array}\right] \text {, }
\end{aligned}
$$




$$
\begin{aligned}
& E(z)=R(z)-Y(z)= \\
& =\left[\begin{array}{c}
\frac{1}{1+G_{\mathrm{P}}(z) G_{\mathrm{C}}(z)} \\
\frac{-G_{\mathrm{P}}(z)}{1+G_{\mathrm{P}}(z) G_{\mathrm{C}}(z)} \\
\frac{-G_{\mathrm{P}}(z)}{1+G_{\mathrm{P}}(z) G_{\mathrm{C}}(z)} \boldsymbol{G}_{0 \mathrm{C}}^{\mathrm{T}}(z) \\
\frac{-1}{1+G_{\mathrm{P}}(z) G_{\mathrm{C}}(z)} \boldsymbol{G}_{0 \mathrm{P}}^{\mathrm{T}}(z)
\end{array}\right]^{\mathrm{T}} \cdot\left[\begin{array}{c}
\boldsymbol{I}(z) \\
\boldsymbol{c}_{0 C} \\
\boldsymbol{c}_{0 P}
\end{array}\right] .
\end{aligned}
$$

From Eq. (17) follows the equivalent full block diagram of the system shown in Fig. 3.

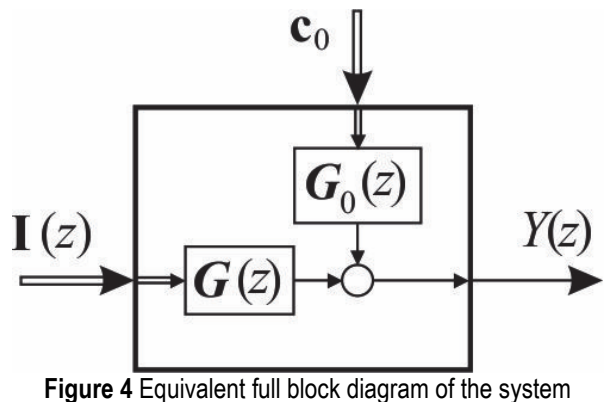

After applying Z-transform including all initial conditions on plant's Eq. (2) we obtain:

$$
\begin{aligned}
& Y(z)=\left[\begin{array}{ll}
G_{\mathrm{P}}(z) & \boldsymbol{G}_{0 \mathrm{P}}(z)
\end{array}\right]\left[\begin{array}{c}
U_{\mathrm{P}}(z) \\
\boldsymbol{c}_{0 \mathrm{P}}
\end{array}\right], \\
& G_{\mathrm{P}}(z)=\frac{1,2417 \cdot 10^{-4} z+1,2125 \cdot 10^{-4}}{z^{2}-1,9311 z+0,9311}, \\
& \boldsymbol{G}_{0 \mathrm{P}}(z)=\left[\begin{array}{c}
\frac{-1,2417 \cdot 10^{-4} z}{z^{2}-1,9311 z+0,9311} \\
\frac{z^{2}-1,9311 z}{z^{2}-1,9311 z+0,9311} \\
\frac{z}{z^{2}-1,9311 z+0,9311}
\end{array}\right]^{\mathrm{T}}, \boldsymbol{c}_{0 \mathrm{P}}=\left[\begin{array}{c}
u_{\mathrm{P}}(0) \\
y(0) \\
y(1)
\end{array}\right] .
\end{aligned}
$$

After applying Z-transform including all initial conditions on controller's Eq. (6) we obtain:

$$
\begin{aligned}
& U_{\mathrm{P}}(z)=\left[\begin{array}{ll}
G_{\mathrm{C}}(z) & \boldsymbol{G}_{0 \mathrm{C}}(z)
\end{array}\right]\left[\begin{array}{c}
E(z) \\
\boldsymbol{c}_{0 \mathrm{C}}
\end{array}\right], \\
& G_{\mathrm{C}}(z)=\frac{\beta z+0,002 \alpha-\beta}{z-0,998}, \\
& \boldsymbol{G}_{0 \mathrm{C}}(z)=\left[\begin{array}{c}
\frac{-\beta z}{z-0,998} \\
\left.\frac{z}{z-0,998}\right]^{\mathrm{T}}, \boldsymbol{c}_{0 \mathrm{C}}=\left[\begin{array}{c}
\varepsilon(0) \\
u_{\mathrm{P}}(0)
\end{array}\right],
\end{array}\right.
\end{aligned}
$$

where $\varepsilon(0)=r(0)-y(0)$.

Using Eqs. (18), (19) and (20) we obtain:

$$
E(z)=\left[\begin{array}{c}
\frac{q_{2} q_{4}}{q_{1} q_{3}+q_{2} q_{4}} \\
\frac{q_{1} \beta z}{q_{1} q_{3}+q_{2} q_{4}} \\
\frac{-z q_{1}}{q_{1} q_{3}+q_{2} q_{4}} \\
\frac{1,2417 \cdot 10^{-4} z q_{2}}{q_{1} q_{3}+q_{2} q_{4}} \\
\frac{\left(-z^{2}+1,9311 z\right) q_{2}}{q_{1} q_{3}+q_{2} q_{4}} \\
\frac{-z q_{2}}{q_{1} q_{3}+q_{2} q_{4}}
\end{array}\right]^{\mathrm{T}} \cdot\left[\begin{array}{c}
R(z) \\
\varepsilon(0) \\
u_{\mathrm{P}}(0) \\
u_{\mathrm{P}}(0) \\
y(0) \\
y(1)
\end{array}\right],
$$

$q_{1}=1,2417 \cdot 10^{-4} z+1,2125 \cdot 10^{-4}$,

$q_{2}=z-0,998$,

$q_{3}=\beta z+0,002 \alpha-\beta$,

$q_{4}=z^{2}-1,9311 z+0,9311$.

Performance index can be calculated as follows,

$I=\sum_{r=1}^{n} \operatorname{Res}\left[E(z) E\left(z^{-1}\right) z^{-1}\right]_{\mid z=z_{r}^{*}}$,

where $z_{r}^{*}$ is root from $z^{-1} E(z)$.

Initial nonzero conditions were identified at the end of a 1,27 $\mathrm{s}$ long time interval during which the servo motor is driven in open loop by voltage $U=1,6 \mathrm{~V}$. These initial conditions are repeatedly achieved whenever the experiment is carried out in the same way. Using the nonzero initial conditions: $u_{\mathrm{P}}(0)=0,1, y(0)=0,2$, $y(1)=0,205, r(0)=0$, the performance index values were calculated for 2577 points, but only some of them were applied to the constant damping coefficient curve, as shown in Fig. 5.

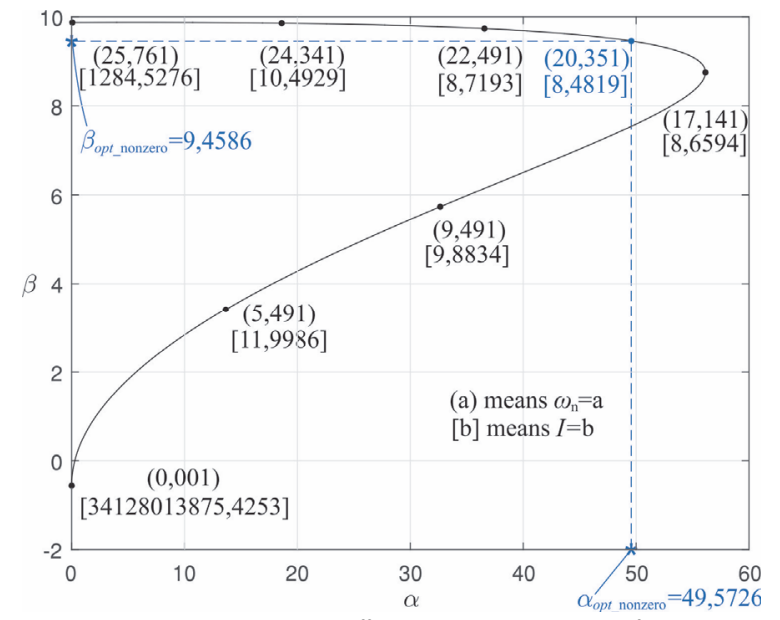

Figure 5 The constant damping coefficient curve with some performance index values applied to it for nonzero initial conditions. Optimal values of the parameters $\alpha$ and $\beta$ are $\alpha_{\text {opt_nonzero }}=49,5726, \beta_{\text {opt_nonzero }}=9,4586$

The sample of the optimal values of parameters $\alpha, \beta$, which gives the optimal minimal value of the performance 
index $I_{\text {min_nonzero }}=8,4819$, is as follows: $\alpha_{\text {opt_nonzero }}=$ $49,5726, \beta_{\text {opt_nonzero }}=9,4586$.

To highlight the difference between the new and the classical approach, the performance index values were also calculated for 2577 points using the zero initial conditions.

Some of these values were applied to the constant damping coefficient curve, as shown in Fig. 6. The optimal values of the parameters $\alpha$ and $\beta$, which in this case give the optimal value of the performance index

$I_{\text {min_zero }}=10,5791$, are as follows: $\alpha_{\text {opt_zero }}=11,0438$, $\beta_{\text {opt_zero }}=9,8749$.

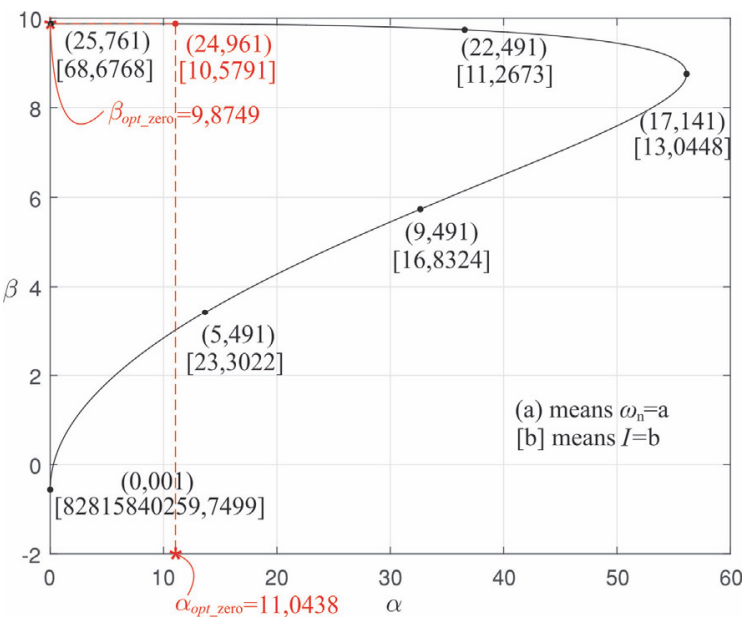

Figure 6 The constant damping coefficient curve with some performance index values applied to it for zero initial conditions. Optimal values of the parameters $\alpha$ and $\beta$ are $\alpha_{\text {opt_zero }}=11,0439, \beta_{\text {opt_zero }}=9,8749$

Experimental results are given for the servo motor closed loop control system functioning with the optimal $\alpha$, $\beta$ parameter values.

The system experimental 0,7 step responses for nonzero and zero initial conditions, that is, for the new theory and for the classical theory, respectively, are shown in Fig. 7.

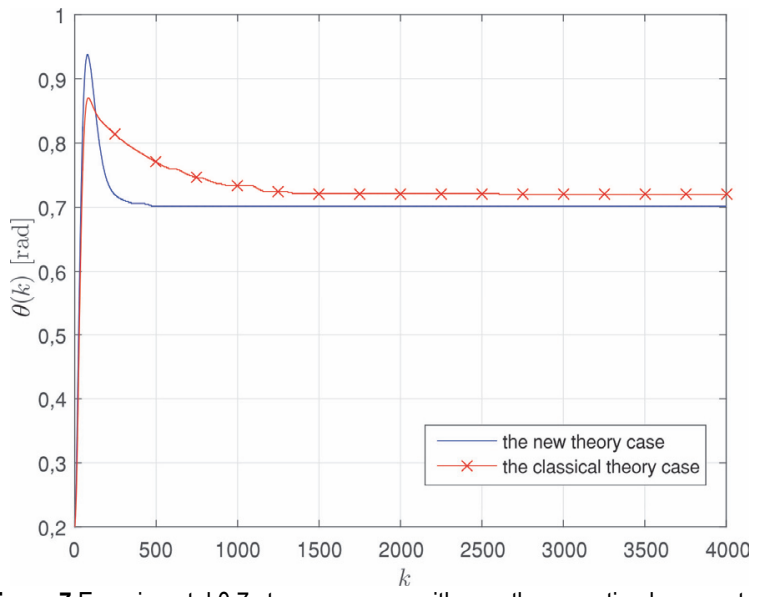

Figure 7 Experimental 0,7 step responses with new theory optimal parameters (blue-starting from nonzero initial conditions) and classical theory optimal parameters (red-starting from nonzero initial conditions)

At first, servo motor works in open loop to get nonzero initial conditions. After that, as soon as they are achieved the system is switched to the closed loop operational mode.

The "red" system experimental response is obtained by the classical theory optimal parameters, "universally optimal" and "valid" for any initial conditions. The "blue" system experimental response is obtained by the new theory optimal parameters valid only for the concrete initial conditions used. Because of that, we started the "red" system response from the same nonzero initial conditions as the "blue" one to make sense in comparing them. It is observable in Fig. 7 that the "red" system response has the tendency to approach the reference signal value later than the "blue" system response. Again, the "blue" system response is much better than that of the "red" one, what promotes the new theory as the only right and appropriate compared to the classical one.

An enlarged segment of Fig. 7 for $0 \leq k \leq 520$ is shown in Fig. 8 to make visible that "blue" and "red" responses start from nonzero initial conditions.

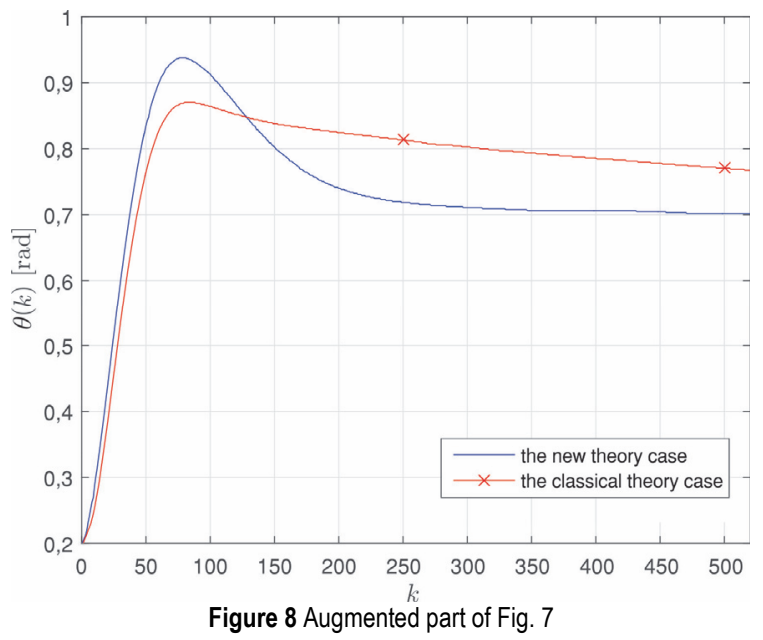

From Fig. 9, which shows the amount of control input given to the plant, it is worth noting that the maximum value of voltage is contained well below $10 \mathrm{~V}$ and it does not reach the saturation level.

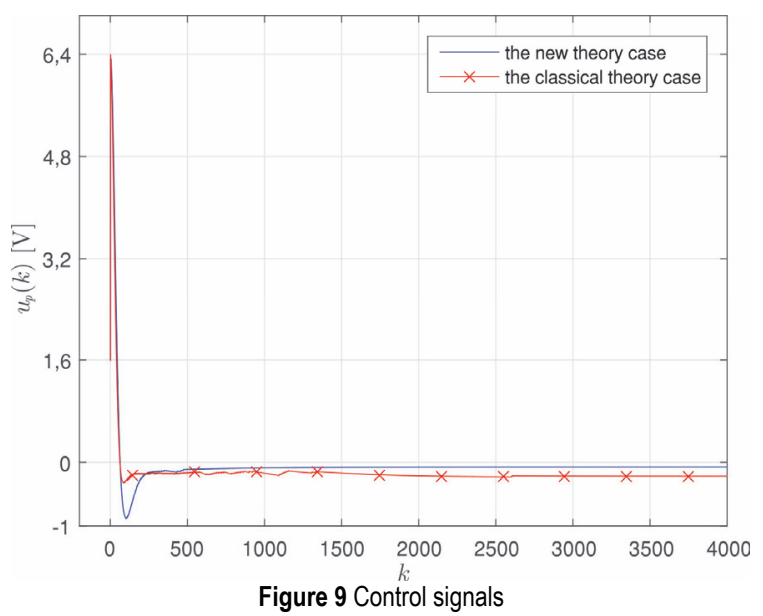

\section{CONCLUSION}

The new conditional optimization procedure of the linear discrete-time systems is discovered and introduced in this paper. The optimization is made in the parameter plane of two unknown and adjustable parameters. Major controversy between the classical transfer function matrix and the system stability investigation using it has been recently solved by developing and introducing the full transfer function matrix $[1,26]$, such that during the system 
conditional optimization the characteristic polynomial of the full transfer function matrix is used but not of the classical transfer function. That is the characteristic polynomial of the so called row nondegenerate full transfer function matrix, the same one as used for the only adequate and appropriate testing of the system stability. A new form of the performance index is introduced that is fully compatible with using the full transfer function matrix. A new, compact calculus $[1,26]$ is also used, without which determining the full transfer function matrix is impossible.

The set of the controller parameters optimal for the control system behavior under all zero initial conditions is not optimal for its operation under nonzero initial conditions. Experimental example is given to show the difference in the system behavior, when it is designed in the classical and the proposed novel way. Effect of initial conditions on behavior of every dynamic system must be taken into account in the process of finding optimal values for controller gains. Each time the system is designed in the classical way and starts from nonzero initial conditions its response is much worse than when the system is designed in new way and starts from nonzero initial conditions too, or when the system is designed in classical way and starts from zero initial conditions. Figs. 5 and 6 illustrate this concluding statement.

PDS (proportional + difference + sum) like controllers are still widely used in industry, in their various forms. Based on the previous experimental results, it is clear that the proposed method can be applied for optimal control of any plant, whose linear mathematical model is known, in the industry that starts operating from nonzero initial conditions.

\section{Acknowledgements}

This work was financially supported by the Ministry of Education, Science and Technological Development of the Serbian Government, under contract 451-03-9/202114/200105, Grant TR-35029 (2021) and Grant TR-35004 (2021).

\section{REFERENCES}

[1] Buchevats, Z. M. \& Gruyitch, Ly. T. (2017). Linear Discrete-Time Systems. Boca Raton, Florida: CRC Press/Taylor \& Francis Group.

[2] Vishnegradski, J. A. (1876). Sur la théorie général des régulateurs. Compres rendusheb dornadaires des séanses de l'Académie des sciences, 83, 318-321.

[3] Neymark, I. (1948). On the values of the parameters for which the feedback control system is stable (in Russian). Avt. Telem., 3 .

[4] Mitrović, D. (1958). Graphical Analysis and Synthesis of Feedback Control Systems I: Theory and Analysis. AIEE Transactions, 77(6), 476-487. https://doi.org/10.1109/TAl.1959.6371486

[5] Mitrović, D. (1958). Graphical Analysis and Synthesis of Feedback Control Systems II: Synthesis. AIEE Transactions, 77(6), 487-496.

[6] Mitrović, D. (1958). Graphical Analysis and Synthesis of Feedback Control Systems III: Sampled-Data Feedback Control Systems. AIEE Transactions, 77(6), 496-502. https://doi.org/10.1109/TAl.1959.6371488
[7] Šiljak, D. D. (1964). Generalization of Mitrovic's method. IEEE Transaction on Applications and Industry, 83(74), 314-320. https://doi.org/10.1109/TAl.1964.5407762

[8] Šiljak, D. D. (1963). Squared error optimization with a relative stability constraint. $\mathrm{PhD}$ Thesis (in Serbian), Belgrade, Yugoslavia: University of Belgrade.

[9] Grujić, Lj. T. (1963). Temperature regulation in heat transfer processes. Diploma work (in Serbian), Belgrade, Yugoslavia: Faculty of Mechanical Engineering.

[10] Śiljak, D. D. (1964). Analysis and synthesis of feedback control systems in the parameter plane I: Linear continuous systems. IEEE Transaction on Applications and Industry, 83(75), 449-458. https://doi.org/10.1109/TAl.1964.5407721

[11] Šiljak, D. D. (1964). Analysis and synthesis of feedback control systems in the parameter plane II: Sampled-Data systems. IEEE Transaction on Applications and Industry, 83(75), 458-466. https://doi.org/10.1109/TAl.1964.5407722

[12] Grujić, Lj. T. (1966). Possibilities of Linear System Design on the Basis of Conditional Optimization in Parameter Plane I: Linear continual systems with constant parameters. Automatika, 1(2), 49-60.

[13] Grujić, Lj. T. (1966). Possibilities of Linear System Design on the Basis of Conditional Optimization in Parameter Plane II: Linear Sampled-Data Systems with Constant Parameters. Automatika, 1(2), 61-71.

[14] Rakić, M. (1965). Contribution to algebraic method for the synthesis of linear systems of automatic control (in SerboCroation). Automatika.

[15] Kalman, R. E. (1958). Optimal synthesis of linear sampling control systems using general performance indexes. Trans. ASME, 80, 1820-1826.

[16] Kalman, R. E. (1960). On the General Theory of Control Systems. IFAC Proceedings Volumes, 1(1), 491-502. https://doi.org/10.1016/S1474-6670(17)70094-8

[17] Xu, H., Datta, A., \& Bhattacharyya, S. P. (2001). Computation of All Stabilizing PID Gains for Digital Control Systems. IEEE Transactions on Automatic Control, 46(4), 647-652. https://doi.org/10.1109/9.917670

[18] Keel, L. H., Rego, J. I., \& Bhattacharyya, S. P. (2003). A New Approach to Digital PID Controller Design. IEEE Transactions on Automatic Control, 48(4), 687-692. https://doi.org/10.1109/TAC.2003.809768

[19] Tantaris, R. N., Keel, L. H., \& Bhattacharyya, S. P. (2003). Stabilization of Discrete-Time Systems by First-Order Controllers. IEEE Transactions on Automatic Control, 48(5), 858-861. https://doi.org/10.1109/TAC.2003.811268

[20] Li, Y., Sheng, A., \& Wang,Y. (2008).Synthesis of PID-type controllers without parametric models: A graphical approach. Energy Conversion and Management, 49(8), 2392-2402. https://doi.org/10.1016/j.enconman.2008.01.017

[21] Gryazina, E. N. \& Polyak, B. T. (2006). Stability regions in the parameter space: D-decomposition revisited. Automatica, 42(1), 13-26. https://doi.org/10.1016/j.automatica.2005.08.010

[22] Gryazina, E. N. \& Polyak, B. T. (2005). Geometry of the stability domain in the parameter space: D-decomposition technique. Proceedings of the $44^{\text {th }}$ ieee conference on decision and control, 6510-6515. https://doi.org/10.1109/CDC.2005.1583206

[23] Padula, F. \& Visioli, A. (2011). Tuning rules for optimal PID and fractional-order PID controllers. Journal of Process Control, 21(1), 69-81. https://doi.org/10.1016/j.jprocont.2010.10.006

[24] Barbosa, R. S. \& Jesus, I. S. (2015). Optimization of Control systems by Cuckoo Search. Controlo2014- Proceedings of the $11^{\text {th }}$ PortugueseConference on Automatic Control, 321, 113-122. https://doi.org/10.1007/978-3-319-10380-8_12

[25] Gruyitch, Ly. T. (2013). Advances in the Linear Dynamic Systems Theory. Tamarac, Florida: Llumina Press. 
[26] Gruyitch, Ly. T. (2017). Linear Continous-Time Systems. Boca Raton, Florida: CRC Press/Taylor \& Francis Group.

[27] Grujić, Lj. T. (1991). Discrete Systems (in Serbian). Belgrade, Yugoslavia: Faculty of Mechanical Engineering.

[28] Gryazina, E. N., Polyak, B. T., \& Tremba, A. A. (2008). Ddecomposition Technique State-of-the-art. Automation and Remote Control, 69(12), 1991-2026. https://doi.org/10.1134/S0005117908120011

[29] Matušů, R. (2011). Calculation of all stabilizing PI and PID controllers. International Journal of Mathematics and Computers in Simulation, 5(3), 224-231.

\section{Contact information:}

Vladimir ZARIĆ, teaching assistant (Corresponding author)

Faculty of Mechanical Engineering

Kraljice Marije 16, 11120 Belgrade 35, Serbia

E-mail: vzaric@mas.bg.ac.rs

Zoran BUČEVAC, full professo

Faculty of Mechanical Engineering

Kraljice Marije 16, 11120 Belgrade 35, Serbia

E-mail: zmbucevac@mas.bg.ac.rs

Radiša JOVANOVIĆ, full professo

Faculty of Mechanical Engineering

Kraljice Marije 16, 11120 Belgrade 35, Serbia

E-mail: rjovanovic@mas.bg.ac.rs 